\title{
Canadian
}

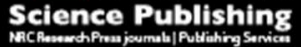

Canadian Journal of Physics

Revue canadienne de physique

\section{ON PHYSICAL APPLICATIONS OF ONE- AND TWO- DIMENSIONAL TOPOLOGICAL OBJECTS WITH FRACTAL DIMENSION}

\begin{tabular}{|r|l|}
\hline Journal: & Canadian Journal of Physics \\
\hline Manuscript ID & cjp-2016-0824.R4 \\
\hline Danuscript Type: & Article \\
\hline Complete List of Authors: & $\begin{array}{l}\text { Gladkov, Sergey; Moskovskij aviacionnyj institut } \\
\text { Bogdanova, Sophie; Moskovskij aviacionnyj institut, } \\
\text { Vestjak, Anatoliy; Moskovskij aviacionnyj institut }\end{array}$ \\
\hline Keyword: & $\begin{array}{l}\text { fractal disc, the resistance force, the Navier-Stokes equation, the Reynolds } \\
\text { number, equation Fourier }\end{array}$ \\
\hline $\begin{array}{r}\text { Is the invited manuscript for } \\
\text { consideration in a Special } \\
\text { Issue? : }\end{array}$ & N/A \\
\hline &
\end{tabular}

SCHOLARONE ${ }^{m}$

Manuscripts 


\title{
ON PHYSICAL APPLICATIONS OF ONE- AND TWO-DIMENSIONAL TOPOLOGICAL OBJECTS WITH FRACTAL DIMENSION
}

\author{
S.O. Gladkov, S.B. Bogdanova, A.V. Vestyak \\ Moscow Aviation Institute (National Research University) (MAI) \\ Volokolamskoe shosse, 4. 125997 Moscow Russia \\ sglad@newmail.ru, sonjaf@list.ru, kaf311@yandex.ru
}

\begin{abstract}
In this paper, the heat transfer coefficient of a body with a fractal surface having the fractal dimension connecting with the fractal dimension of some body, as well as the resistance to a current of fluids flowing normal to such body is shown. It is noted that with an end flow of such flat bodies, by virtue of their fractal perimeter the side resistive force will have the fractal dimension and contribute significantly to the total resistive force acting on any similar structure. It was calculated the dependence of the resistance force of the Reynolds number and was given its graphic illustration. It is noted that the result can be used in medicine in the diagnosis of eye diseases, as well as in marine applications.
\end{abstract}

\section{Introduction}

When taking a quick look at basic physical objects of the world around us, we usually receive our first visual impression from the geometrical shape of such 
objects. Indeed, whichever object we look at, the dimension of any object within our optical perception with $\lambda_{\text {optical }}$ of wave length do not allow to see objects with dimension $\delta<\lambda_{\text {optical }}$. Such task is easily fulfilled by a microscope, and by some zooming in we will be able to see a heavily fractured contour of the geometrical boundary of an object surface rather than an ideal line, even after such surface was mirror smooth finished by some technological processes (mainly polishing). However, even such sophisticated process does not provide smoothing out of all microinhomogeneities of the surface. It means that in any case within the electromagnetic wavelength $\lambda<\xi$ where $\xi$-the linear size of inhomogeneities, the surface will remain rough, though mirror-smooth. We should notice that this simple example shows the imperfection of our world only and prompts to wonder about similar structures with micro in homogeneities seen with the unaided eye, where the boundary of the object being a fractal.

In the process of getting to know the original sources in this direction, for example [1, 2], a very interesting detail emerged. All the papers in this area are devoted to purely mathematical aspects of fractal theory, which ignore the physical properties of the material. That is why the purpose of this article is to close the gap and analyze two purely physical problems (see below). The solution of both problems has a purely physical bias, based on the mathematical apparatus of fractal theory [3]. 
The essence of the study, which will be discussed in this article is as follows. Let us imagine a flat disk with a thickness of $h$ that a stationary flow of a viscous liquid flows perpendicular to its plane. We set ourselves the task of finding out how the resistance force and its heat-conducting properties change if the perimeter of the disk is a series of chaotic roughnesses on the shoreline similarity, but only a closed one (see Fig. 1). In this case, the thickness of the disk $h$ is assumed to be constant along its entire perimeter.

In the case of the classical shoreline, depending on the scale of the measurement, there is an expression different from the whole value, introduced as the fraction limit $\frac{\ln L(l)}{\ln l^{-1}}$, when $l \rightarrow 0$, where $l$ - the scale of the measurement, $L(l)-$ the length of the line in scale $l$. It means that $d_{F}=\lim _{l \rightarrow 0} \frac{\ln L(l)}{\ln l^{-1}} \quad$ or $\quad d_{H}=\lim _{\varepsilon \rightarrow 0} \frac{\ln N(\varepsilon)}{\ln \varepsilon^{-1}}$, which was firstly introduced by Hausdorff $[4,5]$, where $N(\varepsilon)-$ a countable number of linear regions of the partition of the object, $\varepsilon$-the scale of these partitions. This formula "works" not only for the case of self-similar fractal structures, but also for an arbitrary fractal.

As applied to our problem, we will use the modified Minkowski formula, which is most appropriate in our case, but unlike it, we would not cover the surface with circles of radius of $r$, but with equilateral triangles with side. It should be remembered that we are talking about physical laws and real 
geometric bodies in our problem. That is why, in the limiting transition, when $\varepsilon$ tends to zero, we must remember that $\varepsilon \rightarrow a$, where $a$ - some average interatomic distance.

In accordance with the above, for our case we introduce the dimension

$$
d_{S}=\lim _{\varepsilon \rightarrow 0} \frac{\ln N_{S}(\varepsilon)}{\ln S(\varepsilon)}
$$

where $s(\varepsilon)=\frac{\sqrt{3}}{4} \varepsilon^{2}-$ the area. For noncrystalline bodies, a must mean a quantity $a=\left(\frac{V}{N}\right)^{\frac{1}{3}}$, in meaning and in order of magnitude, corresponds to the interatomic distance, $N$ - the total number of atoms in the volume of $V=S h$, where $S$ - the total area of the fractal disk (see below).

We suppose that there is some material body of finite thickness of $h$ with a flat surface and a characteristic linear dimension of $L$. In this case, the macroscopic inhomogeneities of its perimeter $P$ (in the plane of $x-y$ ) could be comparable with $L$ (see Fig. 1). If we introduce an additional fractality of the disk thickness $h$ (see Fig. 1) directed along the axis of $z$, then such objects are called multifractals (see, for example, [1]). They should be understood as a structure that, when stretched in one fixed direction, becomes a one-dimensional thread, and in a perpendicular direction (along the axis $z$ ) will be a "shoreline", which could also be stretched into a one-dimensional thread. However, we should make a 90 degree turn along the first thread. In 
accordance to the formula (1), if we take into account the correction for the dimensionality $\varepsilon$, we get $N_{S}=\left(\frac{\varepsilon}{L}\right)^{-d_{S}}$, and therefore, the area of the figure shown in Fig. 1 can be represented as the following equation:

$$
S=\varepsilon^{-2} N_{S}=\varepsilon^{-2}\left(\frac{\varepsilon}{L}\right)^{d_{S}}=S_{0} \Delta^{d_{S}-2}
$$

where $\Delta=\frac{\varepsilon}{L}$ - the dimensionless parameter and $S_{0}=L^{2}$. Thus, for a plane fractal figure, its area should be described by the dependence (2). This formula would be used by us below in the process of solving two specific physical problems. We should start with the first task.

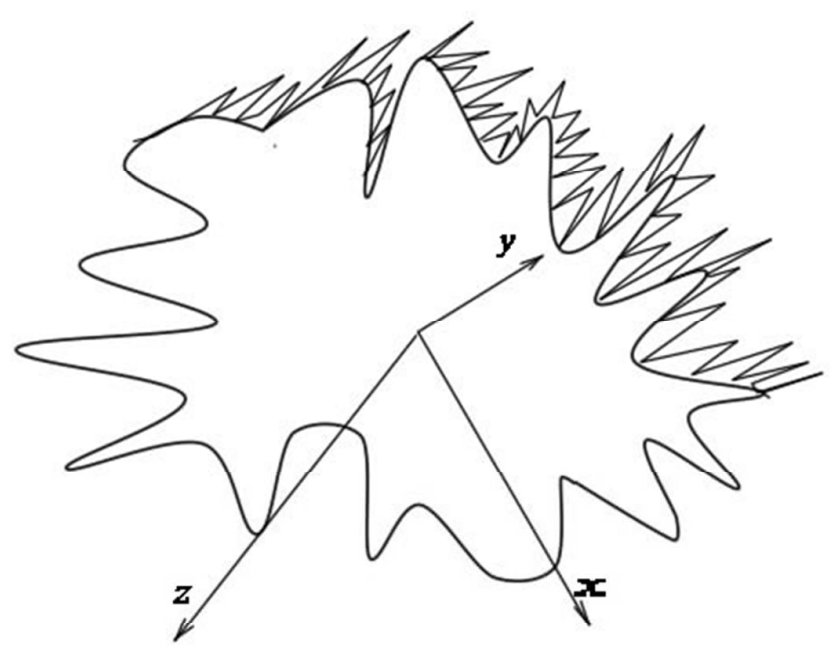

Fig.1 Coordinates $x-y$ lie in the plane of the disc, axis $z$ is perpendicular to the disc plane and extends to the direction of the side fractal surface. 


\section{Heat Transfer Coefficient}

In linear approximation per temperature gradient $\nabla T$, the heat current $\mathbf{q}$ could be presented in accordance with the Fourier formula $\mathbf{q}=-\kappa \nabla T$, where $\kappa-$ a coefficient of heat transfer. In the finite difference form it could be formulated as $q=\kappa \frac{\left(T-T_{0}\right)}{\delta}$, where $\delta-$ a characteristic size of area of heat transfer in the area adjacent to the surface of the body, and $T_{0}$ - the temperature of the thermostat. If the coefficient of heat transfer is presented as $\alpha=S q=\kappa S \frac{\left(T-T_{0}\right)}{\delta}$ and, based on the physical sense, considered to be equal to change of energy per a time unit, the formula (2) would help us obtain the fractal dependence of coefficient of heat transfer having with dimension $\gamma=2-d_{S}$. As a matter of cat, we get the following one:

$$
\alpha=\alpha_{0} \Delta^{d_{s}-2},
$$

where $\alpha_{0}=\kappa S_{0} \frac{T-T_{0}}{\delta}$.

\section{Viscous Flow Resistance}

As for the force resisted by this particular flat object while moving through a viscous fluid in a direction perpendicular to its fractal plain; it is easy to measure based on the following simple considerations. In fact, the surface of a topologically two-dimensional fractal plain travelling through a viscous flow 
is exposed to a pressure equal to $\frac{\rho u^{2}}{2}$, where $\mathbf{u}$-the speed of the flow perpendicular to its plain at large distances. For this reason, aside from purely viscous component of the resistive force, which is known to be [6], in the case of a disc with $R$ radius, we get the following expression:

$$
F_{1}=16 \eta R u
$$

the object must also overcome the pressure of the incoming flow, so we get this equation:

$$
F_{2}=\frac{\rho u^{2}}{2} S
$$

Therefore, the total force should be a sum of those two forces, i.e. it would be determined by the following sum:

$$
F=\frac{\rho u^{2}}{2} S+16 \eta u \sqrt{S}
$$

where $R$ was replaced with $\sqrt{S}$ by an order of magnitude.

The eq. (6) means that the full of resistance force include the both of the viscous force and force of the flux pressure.

From comparing of the summands of (6) it could be seen that with speeds $u<\frac{v}{L}$ (low Reynolds numbers) the force $F_{1}$ would prevail, whereas with $u>\frac{v}{L}$ (high Reynolds numbers), the force $F_{2}$. For this reason, within the real-world 
speed range, when the Reynolds number is high, $u>\frac{v}{L}$ being similar to (3) and we could write:

$$
F \approx F_{2}=F_{02} \Delta^{d_{s}-2},
$$

where $F_{02}=\frac{\rho u^{2}}{2} S_{0}$. When the speeds are low $\left(u<\frac{v}{L}\right.$; we should note that these speeds are close to zero), the formula (6) implies that:

$$
F \approx F_{1}=F_{01} \Delta^{0.5 d_{s}-1}
$$

where $F_{01}=16 \eta u \sqrt{S_{0}}$. When calculating resisting force acting on such fractal flat bodies during motion in viscous fluid, it is necessary to consider one more side resisting force acting on sinuous edge of the body, which, due to its relatively big length exceeding the length of circumference $2 \pi L$, would produce one more significant contribution to the expression (6). Such contribution could be measured in the following manner. We should solve an initial problem about finding the resisting force to be overcome by a $l$ long cylinder streamlined axially with a constant speed $\mathbf{u}$ by a flow of viscous fluid. According to general hydrodynamics principles [6] the resisting force would be presented as follow ones:

$$
F_{i}=\eta \int_{S}\left(V_{i, k}+V_{k, i}\right) d S^{k}-\int_{S} P g_{i k} d S^{k},
$$


where $\quad V_{i, k}=\frac{\partial V_{i}}{\partial x^{k}}-\Gamma_{i k l} V^{l}-$ covariant derivation, $\quad \Gamma_{i k l}=\frac{1}{2}\left(\frac{\partial g_{i k}}{\partial x^{l}}+\frac{\partial g_{l i}}{\partial x^{k}}-\frac{\partial g_{k l}}{\partial x^{i}}\right)-$

Christoffel symbol, and the subscripts and superscripts indicate contravariant and covariant projections of vectors correspondingly, $d S^{k}$-contravariant projection of an element of surface, $g_{i k}-$ covariant components of metrical tensor, and $P$ - pressure near the surface of the cylinder. In a cylindrical coordinate system with axis $z$ directed along the axis of the cylinder, when an element of length could be presented as $d l^{2}=d r^{2}+r^{2} d \varphi^{2}+d z^{2}$, when covariant components of the metric tensor are $g_{r r}=1, g_{\varphi \varphi}=r^{2}, g_{z z}=1$, and contravariant ones are $g^{r r}=1, g^{\varphi \varphi}=\frac{1}{r^{2}}, g^{z z}=1$ correspondingly, the element of surface would have the only non-zero component $d \mathbf{S}=\left(d S^{r}, 0,0\right)$, where $d S^{r}=r d \varphi d z$. As a result, the formula (9) could be transformed into the following:

$$
F_{z}=\eta \int_{S}\left(V_{i, k}+V_{k, i}\right) d S^{k}-\int_{S} P g_{i k} d S^{k}=\left.\eta R \int_{0}^{l} d z \int_{0}^{2 \pi} d \varphi\left(\frac{\partial V_{z}}{\partial r}-\Gamma_{z r z} V^{z}-\Gamma_{r z z} V^{z}\right)\right|_{r=R}-\int_{R}^{\infty} r d r \int_{0}^{2 \pi} P d \varphi
$$

In this case, it was taken into consideration that the speed has the only nonzero component $V_{z}$, directed axially in the line of the streamlining flow. Hence, $\mathbf{V}=\left(0,0, V_{z}(r)\right)$. From definition of Christoffel symbols it is easy to derive that $\Gamma_{r z z}=\Gamma_{z r z}=0$. For this reason, the formula (10) gives the desired expression of the resisting force: 


$$
F_{z}=\left.\eta R \int_{0}^{l} d z \int_{0}^{2 \pi} d \varphi \frac{\partial V_{z}}{\partial r}\right|_{r=R}-\left.2 \pi R l P\right|_{r=R}=\left.2 \pi \eta R l \frac{\partial V_{z}}{\partial r}\right|_{r=R}-2 \pi \int_{R}^{\infty} r P d r .
$$

What remains is to find the dependence between $V_{z}(r)$ and $P(r)$.

According to steady-state Navier-Stokes equation, in case with low Reynolds numbers when a substantial derivative could be disregarded, we get the following one:

$$
\nabla P=\eta \cdot \Delta \mathbf{V}
$$

By taking operation rot from both parts of this equation, we could find that:

$$
\Delta(\operatorname{rot} \mathbf{V})=0
$$

The solution of this equation in form shown in [6] would be:

$$
\mathbf{V}=\mathbf{u}+\operatorname{rot}(\operatorname{rot}(f \cdot \mathbf{u}))
$$

where the function $f$ is yet to be found. After simple transformation and substitution of solution (14) to the equation (13), we could find that the desired function must satisfy a biharmonic equation, i.e.:

$$
\Delta^{2} f=0
$$

In a cylindrical coordinates system, assuming, due to axial symmetry of the problem, that $f=f(r)$, the expression (15) results into:

$$
\frac{1}{r} \frac{\partial}{\partial r} r \frac{\partial}{\partial r} \frac{1}{r} \frac{\partial}{\partial r} r \frac{\partial f}{\partial r}=0
$$


After simple quadruple integration this implies that:

$$
f=C_{1}+C_{2} r^{2}+C_{3} \ln r+C_{4} r^{2} \ln r
$$

It is obvious that a solution could have a physical meaning only when $C_{1}=C_{4}=0$. For this reason, introducing $a=C_{2}, b=C_{3}$ would imply the following one:

$$
f=a r^{2}+b \ln r .
$$

Now substitution of (16) into (14) would show that distribution of speeds near the surface of the cylinder would comply with the dependence, i.e.:

$$
\mathbf{V}=\mathbf{u} \cdot\left(1+\frac{f^{\prime}}{r}\right)+\frac{(\mathbf{u} \cdot \mathbf{r}) \cdot \mathbf{r}}{r}\left(\frac{f^{\prime}}{r}\right)^{\prime}-\Delta(f \mathbf{u}) .
$$

where prime means differentiation by $r$. Since the speed of flow $\mathbf{u}$ is orthogonal to the radial radius-vector $\mathbf{r}$ the second summand is vanishing here and the result would be:

$$
\mathbf{V}=\mathbf{u} \cdot\left(1+\frac{f^{\prime}}{r}\right)-\Delta(f \mathbf{u})
$$

We are interested in an axially directed projection of the speed, therefore it is resulting into the following one:

$$
V_{z}=u\left(1+\frac{f^{\prime}}{r}\right)-(\Delta(f \mathbf{u}))_{z}
$$


In accordance with definition of Laplacian operator acting on a vector function in a curvilinear coordinate system it is possible to write in the following form:

$(\Delta \mathbf{A})^{i}=\Delta A^{i}+\left(\frac{\partial \Gamma_{k l}^{i}}{\partial x^{k}}+\Gamma_{k p}^{i} \Gamma_{k l}^{p}-\Gamma_{k k}^{n} \Gamma_{n l}^{i}\right) A^{l}+2 \Gamma_{k l}^{i} \frac{\partial A^{l}}{\partial x^{k}}-\Gamma_{k k}^{n} \frac{\partial A^{i}}{\partial x^{n}}$. Since the speed of flow $\mathrm{u}$ is orthogonal to the radial radius-vector $\mathrm{r}$ the second summand is vanishing here and the result would be:

$$
(\Delta(f \mathbf{u}))^{z}=u \Delta f
$$

because $\Gamma_{z z}^{r}=g^{r r} \Gamma_{r z z}=\Gamma_{r z}^{z}=g^{z z} \Gamma_{z r z}=0$. Therefore, the (19) leads to the following solution:

$$
V_{z}=u\left(1+\frac{f^{\prime}}{r}\right)-u \Delta f
$$

Or, considering the (16) would be presented in his form:

$$
V_{z}=u\left(1+2 a+\frac{b}{r^{2}}-\frac{1}{r} \frac{\partial}{\partial r} r \frac{\partial}{\partial r}\left(a r^{2}+b \ln r\right)\right)=u\left(1-2 a+\frac{b}{r^{2}}\right)
$$

From the boundary condition $\left.V_{z}\right|_{r=R}=0$ and the condition $V_{z} \rightarrow u$ when $r \rightarrow \infty$ as a result we have two decisions $a=0, b=R^{2}$. Therefore, the function would be:

$$
f=R^{2} \ln r,
$$

and the speed would be: 


$$
V_{z}=u\left(1-\frac{R^{2}}{r^{2}}\right)
$$

As we could see from the expression of (11) we need to know the dependence $P(r)$. To calculate the dependence of the pressure as a function of radial coordinate $r$ in accordance with the equation (12), take into account the equation (15), we could have the following result:

$$
\begin{aligned}
& \nabla P=\eta \cdot \Delta(\operatorname{rot}(\operatorname{rot}(f \mathbf{u})))=\eta \cdot \Delta(\operatorname{grad}(\operatorname{div}(f \mathbf{u}))-\Delta(f \mathbf{u}))= \\
& =\eta \cdot \Delta(\operatorname{grad}(\operatorname{div}(f \mathbf{u})))-\eta \cdot \mathbf{u} \cdot\left(\Delta^{2} f\right)=\eta \cdot \nabla(\Delta(\operatorname{div}(f \mathbf{u})))
\end{aligned} .
$$

Therefore, by "taking out" the gradients from left and right we would find that:

$$
P=P_{0}+\eta \cdot \Delta \operatorname{div}(f \mathbf{u})
$$

where $P_{0}$ - external constant pressure. This will immediately lead us to the next result, i.e.:

$$
P=P_{0}+\eta \cdot \Delta \operatorname{div}(f \mathbf{u})=P_{0}+\eta \cdot \Delta(\mathbf{u} \cdot \nabla f)=P_{0}+\eta \cdot \Delta\left(\frac{\mathbf{u} \cdot \mathbf{r}}{r} f^{\prime}\right)=P_{0},
$$

where it was taken into consideration that $\mathbf{u} \cdot \mathbf{r}=0$. Therefore, by substituting the (24) and (22) into the formula (11) we would find the following formula for the resisting force, i.e.:

$$
F_{z}=4 \pi \eta l u
$$


While finding (25), we took into consideration that there was no pressure on the cylinder surface, whereas on infinity the pressure is $P_{0}$. We should notice that the solution of the problem on dependence of the resisting force on the cylinder's radius with flow moving crosswise was presented in the paper [7], which was not done in neither [6] and no [8-10].

In this case of a thin flat object the parameter $l$ must be replaced for the thickness of a fractal disc $h$. Hence,

$$
F_{z}=4 \pi \eta u h,
$$

Therefore, according to (10) the total resisting force is a sum, i.e.:

$$
F=\frac{\rho u^{2}}{2} S+16 \eta u \sqrt{S}+4 \pi \eta u h .
$$

As it implies, the perimeter's being fractal is not important here. In the passage to the limit, when $h \rightarrow 0$, we could substitute parameter $\varepsilon$ for $h$ in the formula (2), i.e. to consider them dependent. If it is assumed, the resisting force would well have physical form where the fractal dependence of the resisting force on dimension could be observed. In fact,

$$
S=S_{0}\left(\frac{h}{L}\right)^{d_{s}-2},
$$

and the (27) is transformed into the following one:

$$
F=\frac{\rho u^{2}}{2} S_{0}\left(\frac{h}{L}\right)^{d_{s}-2}+16 \eta u \sqrt{S_{0}}\left(\frac{h}{L}\right)^{0.5 d_{s}-1}+4 \pi \eta u h .
$$


As we could see, when $h \rightarrow 0$, the last summand could be put aside (we should notice that $d_{S}>1$ ) and the formula would become simpler:

$$
F=\frac{\rho u^{2}}{2} S_{0}\left(\frac{h}{L}\right)^{d_{S}-2}+16 \eta u \sqrt{S_{0}}\left(\frac{h}{L}\right)^{0.5 d_{S}-1}
$$

The resulting expression lacks one more summand relating to pressure on the side edge. One can be found through the following simple reasoning. It is clear that the $h$ thick side surface is exposed to force $\Delta F=P \Delta \sigma=\frac{\rho u^{2}}{2} h \Delta l$, where $\Delta l$-small length of the closed fractal line. We should substitute it for $\Delta l=h\left(\frac{h}{L}\right)^{-d_{F}}$, where the index of power $d_{F}$ is a coastline-type fractal dimension which is closed within the observed object of finite linear size $L$. As a result, the side resistance would be the following:

$$
\Delta F=\frac{\rho u^{2}}{2} h^{2}\left(\frac{h}{L}\right)^{-d_{F}}=\frac{\rho u^{2}}{2} S_{0}\left(\frac{h}{L}\right)^{2-d_{F}}
$$

What is more, we should notice that though this does not include the viscosity of the environment, the force $\Delta F$, however, is a quite real force acting on this particular fractal object. In principle, it is obvious enough, because low speeds of flow could be disregarded comparing with the linear resisting force (it results from substantial derivate in the Navier-Stokes equation). Whereas, when it comes to high Reynolds numbers (i.e. with relatively high speeds) the resisting force, as known from [11], would be proportional to the square of 
the speed. In this particular case, with a fractal object, a big importance is attached to the first summand in the formula (29) because even at low speeds its contribution would be extremely important. Also, by adding $\Delta F$ according to (30) into the general expression (29), we would find the desired resistance, i.e.:

$$
F=\frac{\rho u^{2}}{2} S_{0}\left(\frac{h}{L}\right)^{d_{S}-2}+16 \eta u \sqrt{S_{0}}\left(\frac{h}{L}\right)^{0.5 d_{s}-1}+\frac{\rho u^{2}}{2} S_{0}\left(\frac{h}{L}\right)^{2-d_{F}} .
$$

As can be seen, this expression should have an extremum for argument $x=\frac{h}{L}$. If nondimensionalized and written in relative units, the formula (31) would be presented in the following form:

$$
f(x)=\frac{2 F}{\rho u^{2} S_{0}}=x^{d_{s}-2}+x^{2-d_{F}}+b x^{0.5 d_{s}-1},
$$

$b=\frac{32 v}{u \sqrt{S_{0}}}$. Below the Figures 2-4 depict two-dimensional dependences of resisting force on the coordinate $x$ and fractal dimension $d_{F}$ with different parameters $b=\frac{32 v}{u \sqrt{S_{0}}}=\frac{32}{\operatorname{Re}}$, where $\operatorname{Re}=\frac{u \sqrt{S_{0}}}{v}-$ is a Reynolds number. 


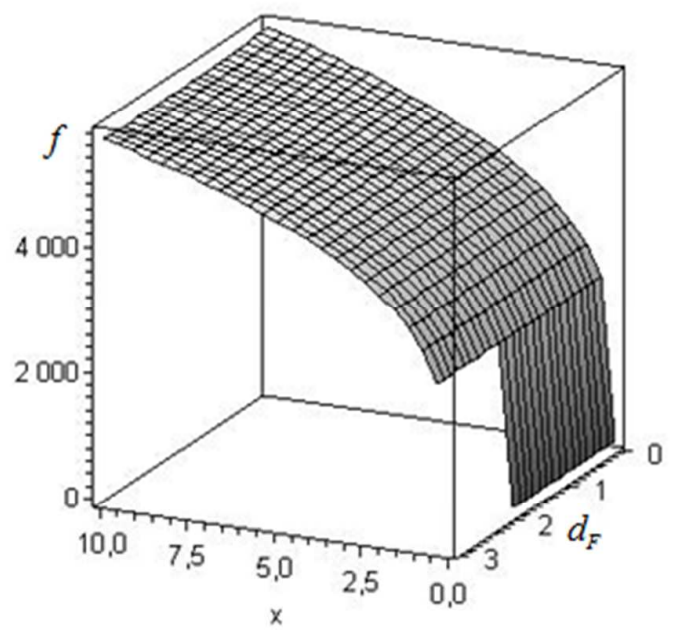

Fig.2. It is shown the dependence of the resisting force $f$ in accordance to the (32) on current coordinate $x$ and on the fractal dimensions $d_{F}$. The graph was obtained with

$$
\mathrm{Re}=0.01
$$

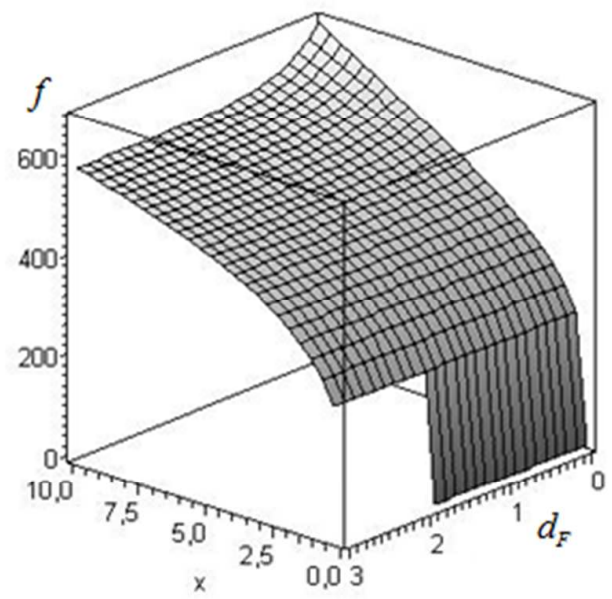

Fig.3. It's shown the dependence of the resisting force $f$ in accordance to the (32) on current coordinate $x$ and on the fractal dimensions $d_{F}$. The graph was obtained with

$$
\operatorname{Re}=0.1
$$




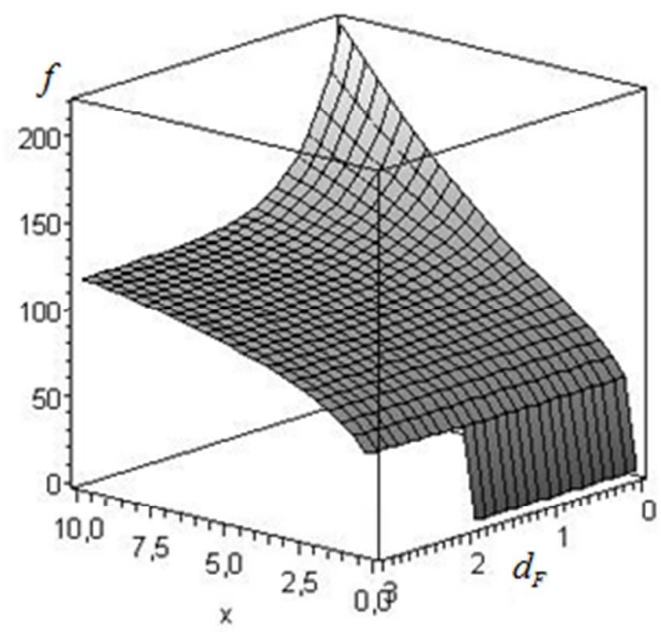

Fig.4. It's shown the dependence of the resisting force $f$ in accordance to the (32) on current coordinate $x$ and on the fractal dimensions $d_{F}$. The graph was obtained with

$$
\mathrm{Re}=0.5
$$

The graph of dependency of resisting function $f$ is depicted on Fig. $1-4$ with different parameter $b=\frac{32 v}{u \sqrt{S_{0}}}=\frac{32}{\operatorname{Re}}$, where $\operatorname{Re}=\frac{u \sqrt{S_{0}}}{v}-$ Reynolds number.

Comparison with the results of the authors and highlights.

As shown by the analysis of literature sources of [1,2, 7-12], in each of the papers solved its own problem, unrelated to the analysis of the effects of hydrodynamic flow around fractal disks and surfaces. For example, in a huge number of papers, which were shown in $[1,2]$ the main emphasis was on the experimental detection of effects associated with the manifestation of 
fractality of structures, by the way, non-hydrodynamic nature. However, in the paper of [12], it was searched the hydrodynamic flow past rough surfaces for various Reynolds numbers. As it is shown by our theoretical analysis, the qualitative behavior in [12] is in conformity with the results reflected in this article. But, for example, in the paper [7], the effects of hydrodynamic flow past an extended smooth cylindrical object were considered for which the resistance force acting along the surface of the rod was calculated, which was not previously done, but what has been continued in this paper.

Proceeding from the above, we must state that the main achievement of the study is the theoretical prediction and calculation of the resistance force and heat transfer coefficient through the fractal dimension.

\section{Discussions}

To sum up our research work, we would like to pay attention to the following two important moments.

1. In the paper of [12], the experiment was performed to measure the drag force of an aircraft with allowance for the roughness of its surface at different Reynolds numbers. It turned out that the resistance force decreases with increasing Reynolds number and an increase in the difference $2-d_{F}$, which is analytically confirmed in our paper (see formula (31) and Figures 2-4).

2. We would like to take note of the practical significance of our done research. The main thing among the possible practical applications, in our opinion, is the usage of formulas (31) and (32) in ophthalmology. 
Indeed, since the shape of the eye lens is a disc that will eventually wear out, and gradually takes the shape of the fractal object, here we can use the general expression for the calculated contact resistance force (31), (32). On the degree of deviation from the reference value (perfect vision), we could draw conclusions about the degree of lens wear. When the roughness on the surface of the lens is to increase the resistance force, and therefore the pressure in the eye on the retina begin to rise. It means that we could not measure the pressure on the retina, to talk about the weakening of view, to keep watch only the degree of wear. What is more, apart from this application, depending on results can also be used in marine applications, when the degree of wear of the screw vessel will manifest its fractal nature.

Acknowledgments

In conclusion, the authors would like to express sincere gratitude and appreciation to professor S.D. Beneslavskiy from of the MSU M. V. Lomonosov, which given a tumble and helpful advice, accounting which only contributed to the improvement of its quality.

\section{Conclusion}

In the conclusion, we would like note some main results of our research.

1. The fractal dimension of the heat transfer coefficient was found, which, according to (3), is equal to $\lambda=2-d_{S}$, as it is see from (7) 
2. The resistance force, which the cylindrical object undergoes along its longitudinal axis along the axis of symmetry (formula (25)), was found;

3. A general expression was obtained for the resistance force acting on a fractal plane body of small thickness $h$, flowing along a longitudinal or transverse flow of a viscous fluid;

4. A qualitatively correct dependence (31) was noted for any flow velocities.

\section{REFERENCES}

1. Journal of Fractional Calculus and Applications. 2001. V. 1. №1. PP.19. ... 2017. V. 8(2). №20. PP.237-245.

2. Chaos, Solitons \& Fractals. 2009. V.39. PP. 1-2530 ... 2017. V.100. PP.1-110.

3. Mandelbrot B. B. The Fractal Geometry of Nature. N.Y.: W.H.Freeman, 1983.

4. M.Shreder. Fractaly, khaos, stepenniye zakony. Moskva-Izhevsk R\&C Dynamics. 2001. 327p. (Shreder, M., Fractals, chaos, power laws, in Russian).

5. Glensdorf, P., Prigozhin, I., Termodinamicheskaya teoriya struktury, ustoychivosty I fluktuatsiy. M.: Editorial. 2003.280p. (Glensdorf, P., Prigozhin, I., Thermodynamic theory of structure, stability and fluctuations, in Russian) 
6. Landau, L.D., Lifshitz, E.M., Gidrodinamika. T.6. M.: Nauka. 1988. 600 p. (Landau, L.D., Lifshitz, E.M., Hydrodynamics, Volume 6, in Russian)

7. S.O. Gladkov. To the theory of nonlinear dynamic equations for the long elastic rod in viscous media. International Journal of Mathematical Models and Methods in Applied Sciences. 2015. V. 9. PP. 166 - 170.

8. S.O.Gladkov, S.B.Bogdanova. On the Magnetic Susceptibility of Fractal Magnetic Wires. Universal Journal of Physics and Application, Vol. 1(3), 2013. PP. 245 - 248. DOI: 10.13189/ujpa.2013.010305.

9. S.O.Gladkov, S.B.Bogdanova. The heat-transfer theory for quasi-ndimensional system. Physica B: Condensed Matter. 2010. V.405. PP.1973-1975. DOI: 10.1016/j.physb.2010.01.077.

10.S.O.Gladkov, S.B.Bogdanova. To the theory of the longitudinal magnetic susceptibility of quasi-three-dimensional ferromagnetic dielectrics. Physics of the solid state. 2012. V.54. №1. PP.74-78. DOI: $10.1134 / \mathrm{S} 1063783412010131$.

11.Kochin, N.E., Kibel', N.V., Rose, N.V., Teoreticheskaya gidromekhanika. Ch.1,2. M.: Fizmatlit. 1963. (Kochin, N.E., Kibel', N.V., Rose, N.V., Theoretical hydromechanics, in Russian).

12. M. A. Brutyan, V.P.Budayev, A.V.Volkov, A.M.Zhitlukhin, A.V.Karpov, N.S.Klimov, I.S.Men'shov, V.L.Podkovyrov, A.J.Urusov, A.A.Uspenskiy, M.V.Ustinov. The influence of the fractal microstructure of a rigid 
surface on characteristics of a turbulent boundary layer. Scientific notes TsAGI (Central Aerohydrodynamic Institute). 2013. № 4. PP.15-30. 